Curvature-induced D-band Raman scattering in folded graphene

This article has been downloaded from IOPscience. Please scroll down to see the full text article.

2010 J. Phys.: Condens. Matter 22334205

(http://iopscience.iop.org/0953-8984/22/33/334205)

View the table of contents for this issue, or go to the journal homepage for more

Download details:

IP Address: 192.12.184.2

The article was downloaded on 24/08/2010 at 00:10

Please note that terms and conditions apply. 


\title{
Curvature-induced D-band Raman scattering in folded graphene*
}

\author{
Awnish K Gupta ${ }^{1}$, Cristiano Nisoli ${ }^{2}$, Paul E Lammert ${ }^{1}$, \\ Vincent H Crespi ${ }^{1,3,4}$ and Peter C Eklund ${ }^{1,3}$ \\ ${ }^{1}$ Department of Physics, The Pennsylvania State University, University Park, PA 16802, USA \\ 2 Theoretical Division, Los Alamos National Laboratory, Los Alamos, NM 87545, USA \\ ${ }^{3}$ Department of Materials Science and Engineering and Materials Research Institute, \\ The Pennsylvania State University, University Park, PA 16802, USA \\ E-mail: crespi@phys.psu.edu
}

Received 1 June 2010, in final form 14 July 2010

Published 4 August 2010

Online at stacks.iop.org/JPhysCM/22/334205

\begin{abstract}
Micro-Raman scattering from folds in single-layer graphene sheets finds a D-band at the fold for both incommensurate and commensurate folding, while the parent single-layer graphene lacks a D-band. A coupled elastic-continuum/tight-binding calculation suggests that this D-band arises from the spatially inhomogeneous curvature around a fold in a graphene sheet. The polarization dependence of the fold-induced D-band further reveals that the inhomogeneous curvature acts as a very smooth, ideal one-dimensional defect along the folding direction.
\end{abstract}

(Some figures in this article are in colour only in the electronic version)

Single-walled carbon nanotubes (SWCNTs) can be conceptualized as rolled-up graphene ribbons [1, 2] whose electronic and vibrational properties are affected by both curvature and confinement around the circumference. Although confinement has been studied a great deal in SWCNTs [2, 3], graphene nanoribbons [4, 5] and small graphene flakes [6], curvature in the absence of confinement has not, since these two properties are inseparable in a nanotube. Folds in graphene sheets provide a means to decouple curvature from confinement: the fold has curvature but no associated confinement, since the graphene sheet continues on either side of the fold for several microns. Here we demonstrate that the spatially inhomogeneous curvature of a graphene fold generates a Raman D-band of unique origin and character. Unlike the normal D-band of $\mathrm{sp}^{2}$ carbons, it does not require structural disorder. Unlike the abrupt and possibly ragged edge of a graphene flake (which also generates a D-band), a graphene fold is naturally smooth and uniform due to the intactness of the $\mathrm{sp}^{2}$ layer and the long-range nature of the elastic interactions that shape the fold. Hence a fold provides ideal specular scattering in two dimensions. The frequency, intensity and polarization response of the fold-induced D-band

\footnotetext{
* The work described herein constitutes one of the last research projects with Peter's active involvement. We are honored to be able to share authorship with him one last time.

4 Author to whom any correspondence should be addressed.
}

depend on the crystallographic direction along which the sheet folds in a manner consistent with the expectations of doubleresonant scattering for specular scattering from a smooth onedimensional perturbation.

Folded graphene samples were prepared by micromechanical cleavage of highly oriented pyrolytic graphite (HOPG) [7]. Scotch tape (3M) was pressed onto HOPG, removed, and then rubbed onto $\langle 100\rangle$-oriented $\mathrm{Si}$ with a $100 \mathrm{~nm}$ thermal oxide, thereby transferring to the substrate graphene flakes 1-20 layers thick and $1-50 \mu \mathrm{m}^{2}$ in area. These flakes sometimes fold back upon themselves [8-10]. All flakes chosen for Raman study had a large overlap between the top and bottom sides of the fold — at least $15 \mu \mathrm{m}^{2}$ — to avoid sheetedge effects. Atomic force microscopy (AFM) measurements in non-contact mode using standard silicon AFM tips (MFD3D Asylum Research, tips from MakroMasch Inc.) confirm the folding of a single layer onto itself, showing a welldefined plateau, which reveals the number of layers in the flake. Figure 1(a) shows an AFM topography image of a typical fold. The fold is expected to protrude upward from the substrate in a bulb-like shape, as shown schematically in figure 4(a). Bulbs are difficult to see in topography due to the small height difference between the bulb and the flat bilayer, but they are visible in AFM phase-contrast images, since the bulb is softer mechanically than the remainder of the folded layer. Figure 1(b) provides a phase-contrast image of the 

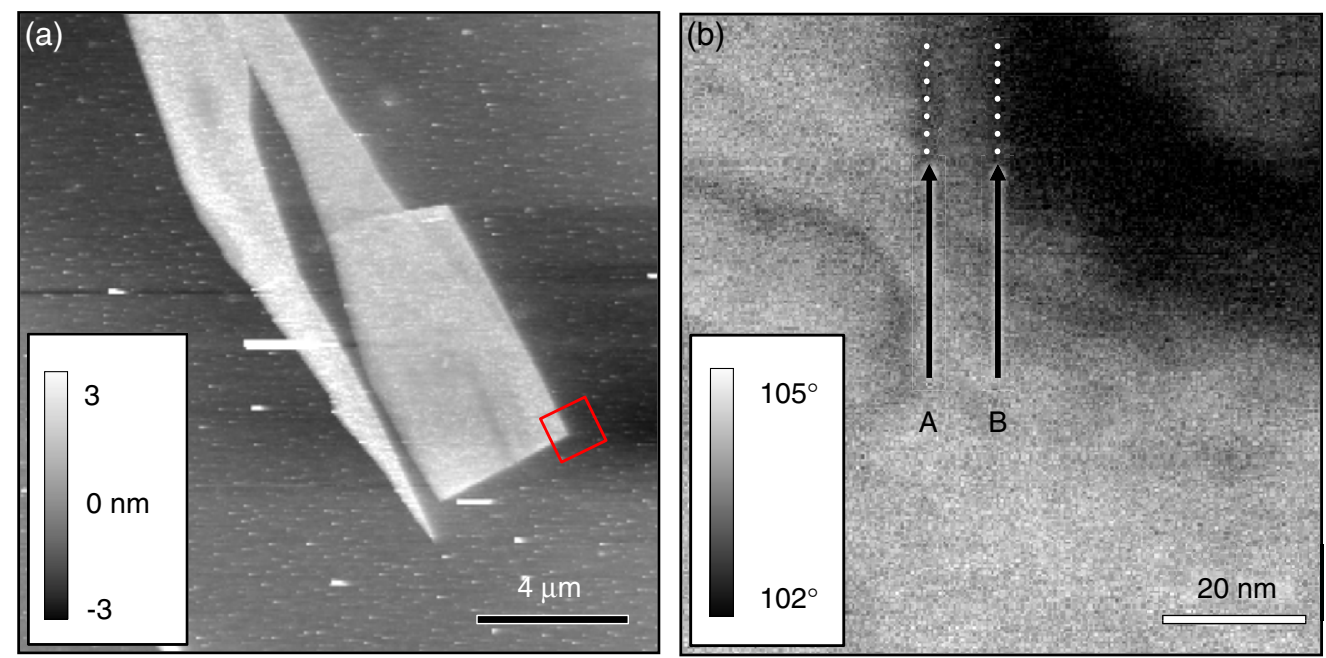

Figure 1. (a) AFM topography image of a folded flake on $\mathrm{SiO}_{2}$. (b) AFM phase image of the fold area indicated by the square in the left-hand image, showing phase contrast from the mechanically soft bulb (the right-hand panel is rotated clockwise by about $120^{\circ}$ with respect to the red square in the left-hand panel). The outer and inner boundaries of the bulb are marked with dotted lines (see figure 4 for a schematic of the bulb). The upper bound on the bulb width is approximately $2 \mathrm{~nm}$ (accounting for an AFM tip width of $\sim 10 \mathrm{~nm}$ ), consistent with the elastic model.

fold marked by the red square in figure 1(a); the dotted lines mark the inner and outer boundaries of the bulb. Since the AFM tip width of $\sim 10 \mathrm{~nm}$ (provided by the manufacturer) is comparable to the observed width of the bulb of $\sim 12 \mathrm{~nm}$, the AFM measurements imply that the bulb is very narrow, consistent with the elastic modeling described below.

The graphene layers across the fold may be either stacked commensurately without interlayer rotation (e.g. AB), or stacked with an interlayer rotation that could be either commensurate (in a long-period super-cell defined by a Moiré pattern) or fully incommensurate (if no point on the rotated lattice coincides precisely with a point on the original). Although most choices of interlayer rotation angles are actually incommensurate, these can lie very close to nearby high-order commensurations and can locally relax into them, elastically. Also, a long-period skewed commensuration and a true interlayer incommensuration are essentially indistinguishable for many physical processes. Hence we denote the non-AB case generically as 'skewed stacking'. Raman experiments [8-10] on bilayer graphene flakes have established distinct Raman signatures for $A B$ and skewed stacking. For example, commensurate stacking produces four clear peaks in the 2D-band $\left(\sim 2700 \mathrm{~cm}^{-1}\right)$ whereas skewed stacking has a single sharp 2D peak. We acquired micro-Raman data using a $100 \times$ objective lens in a Renishaw inVia Raman microscope in backscattering geometry with excitation from a $514.5 \mathrm{~nm}$ Coherent Innova Argon ion laser. Figure 2 shows Raman spectra from 1200 to $3300 \mathrm{~cm}^{-1}$ taken from commensurate and skewed folds (with incident and scattered light polarized parallel to the fold), compared to the spectrum of a single-layer graphene flake. Since the laser spot is much larger than the width of the folded bulb, these spectra include contributions from both the bulb itself and the interior portions of each folded sheet. The spectra are normalized by the G-band intensity and plotted

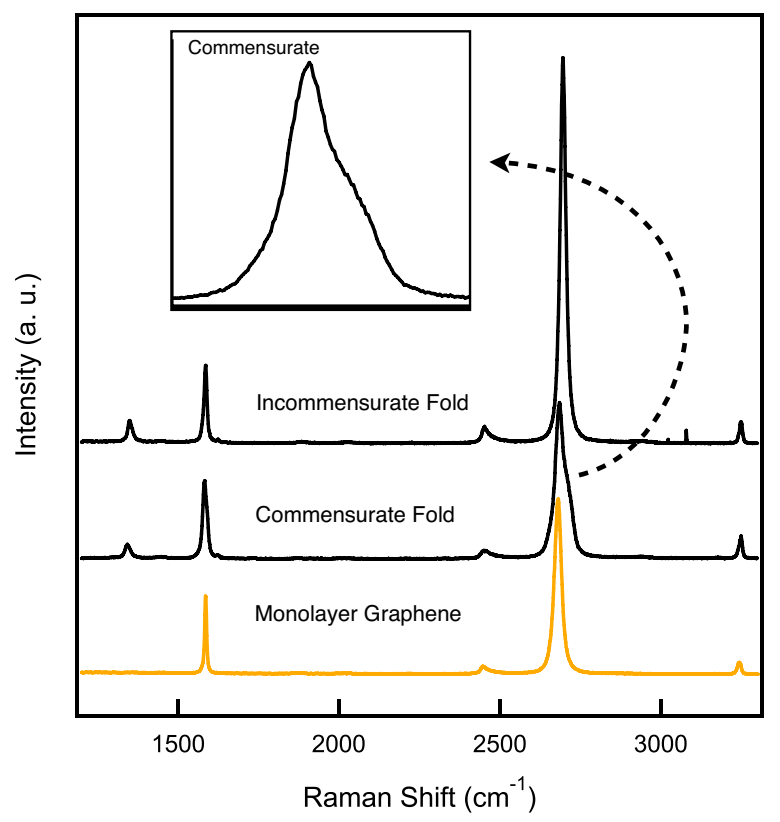

Figure 2. Raman spectra from a fold with skewed or incommensurate stacking between layers, from a commensurate fold, and from a monolayer. Data for folds are collected with the laser spot centered on the fold, as determined by the line scans of figures 3(a) and (b). The inset shows details of the multi-component 2D peak for commensurate folding. Both folds show a Raman feature near $1350 \mathrm{~cm}^{-1}$, the characteristic frequency range of double-resonant D-band scattering, whereas the monolayer does not. Raman spectra were collected using $514.5 \mathrm{~nm}$ laser excitation under ambient conditions.

with a vertical offset for clarity. In all spectra, we see strong peaks at $\sim 1580$ (G-band), $\sim 2450$ (M-K scattering) $\sim 2700$ (2D-band), and $\sim 3250 \mathrm{~cm}^{-1}\left(2 \mathrm{D}^{\prime}\right)$, as reported for graphene flakes [11-13]. However, only the folded sheets show a peak at 

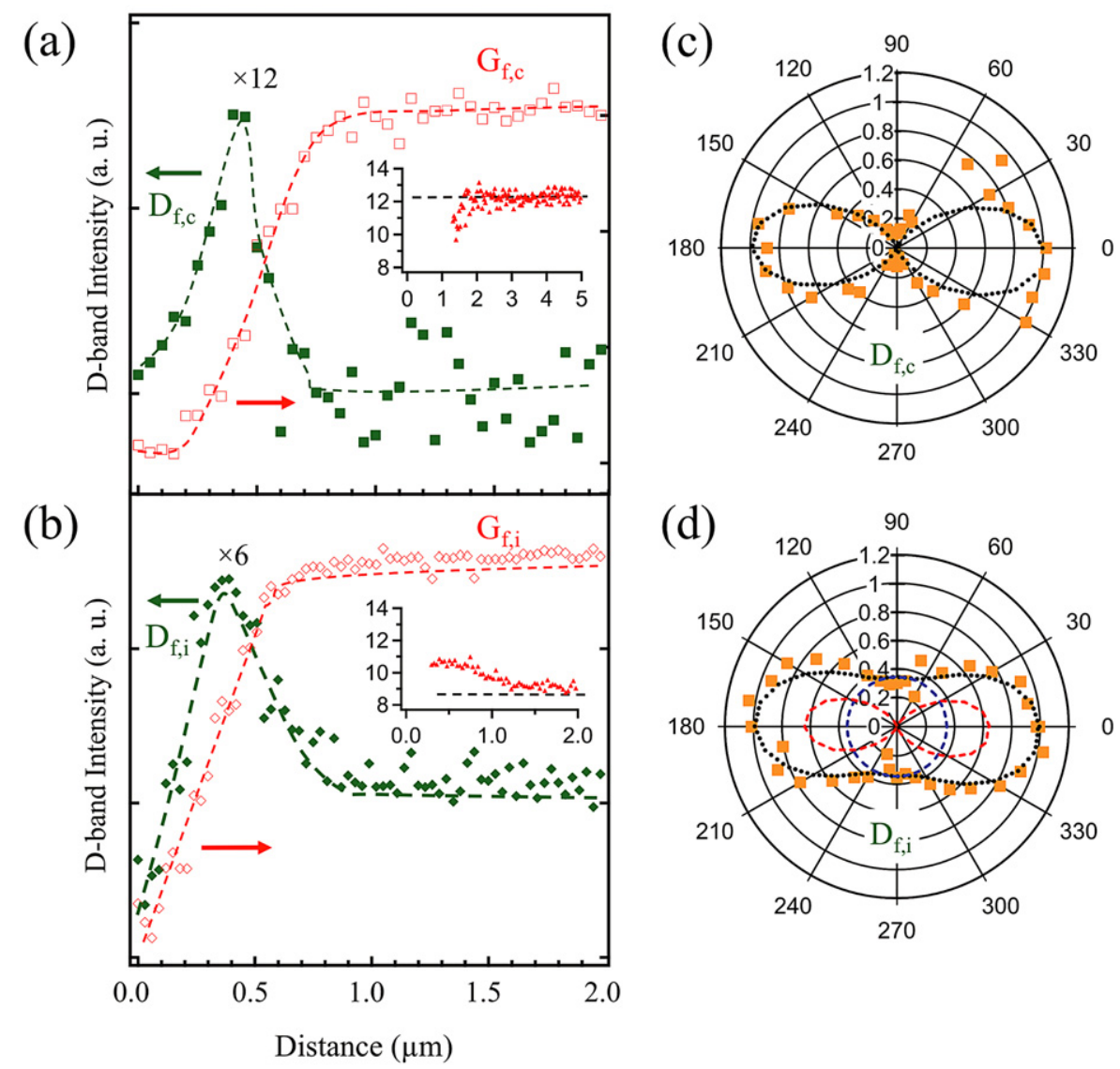

Figure 3. Raman intensity of the D-band (solid symbols) and G-band (open symbols) across commensurate (a) and skewed (or incommensurate) folds (b). The D-band intensity is scaled by the G-band intensity, with table 1 providing the scaling factors. Insets show the G-band linewidths (in $\mathrm{cm}^{-1}$ ) across the fold (in $\mu \mathrm{m}$ ), as discussed in the main text. (c) Polarization dependent D-band intensity for a commensurate fold with least-squares fit to $\cos ^{4} \theta$ (dotted line). (d) Polarization dependent D-band intensity for an incommensurate fold, fitted to a combination of a constant and $\cos ^{4} \theta$ (dashed lines), representing the contributions from the interior-derived I-band and bulb-derived D-band, respectively.

Table 1. Peak-fitting results for the D-band originating from a skewed (or incommensurate) fold, a commensurate fold, and a graphene monolayer.

\begin{tabular}{llll}
\hline & D-band (position in $\mathrm{cm}^{-1}$ ) & Linewidth $\left(\mathrm{cm}^{-1}\right)$ & $I_{\mathrm{D}} / I_{\mathrm{G}}$ ratio \\
\hline Skewed/incommensurate fold & 1351 & 17 & $\sim 0.12^{\mathrm{a}}$ \\
Commensurate fold & 1344 & 20 & $\sim 0.07$ \\
Graphene edge $^{\mathrm{b}}$ & 1344 & 14 & $\sim 0.1-0.22$ \\
\hline
\end{tabular}

${ }^{\mathrm{a}}$ After removing the contribution of I-band scattering from the flake interior. ${ }^{\mathrm{b}}$ From [24].

$\sim 1350 \mathrm{~cm}^{-1}$ (the D-band). We attribute the $\sim 1350 \mathrm{~cm}^{-1}$ peak to the presence of the fold, since it is not seen in any singlelayer regions of the same flake, away from the fold. The inset to figure 2 shows details of the 2D-band near a commensurate fold. Since this spectrum is a superposition of contributions from the bulb and the interior of the commensurately stacked bilayer, it is fitted with two components: a single peak from the bulb and four peaks from the commensurate bilayer. This distinct superposition of features in the 2D-band distinguishes a fold from a sheet edge, since the 2D-band at an edge lacks the bulb-derived component. Distinguishing a fold from an edge is more subtle for a skewed fold. In addition to direct AFM observation of a bulb, folds and edges can be distinguished by the Raman response: when $E_{\text {in }}$ is parallel to the fold or edge and $E_{\text {out }}$ is perpendicular, the D-band response is vanishing for an edge but substantial for a fold, about $40 \%$ of that when $E_{\text {in }}$ and $E_{\text {out }}$ are both parallel to the fold, as shown in figure 3 . Finally, the D-band is fitted with a single least-squares Lorentzian peak; peak-fitting results are summarized in table 1.

To separate fold-derived from interior-derived contributions, we performed Raman line scans across both commensurate and skewed folds, moving the sample stage in $\sim 30-50 \mathrm{~nm}$ steps while maintaining the incident and scattered polarizations parallel to the fold. Figures 3(a) and (b) plot the D-band (integrated from 1300 to $1400 \mathrm{~cm}^{-1}$ ) and G-band (integrated from 1530 to $1630 \mathrm{~cm}^{-1}$ ) intensities and linewidths from Lorentzian least-squares fits. For commensurate folding, the G-band linewidth increases from 9 to $12 \mathrm{~cm}^{-1}$ as one traverses from the fold to the flake interior, as expected for a system 

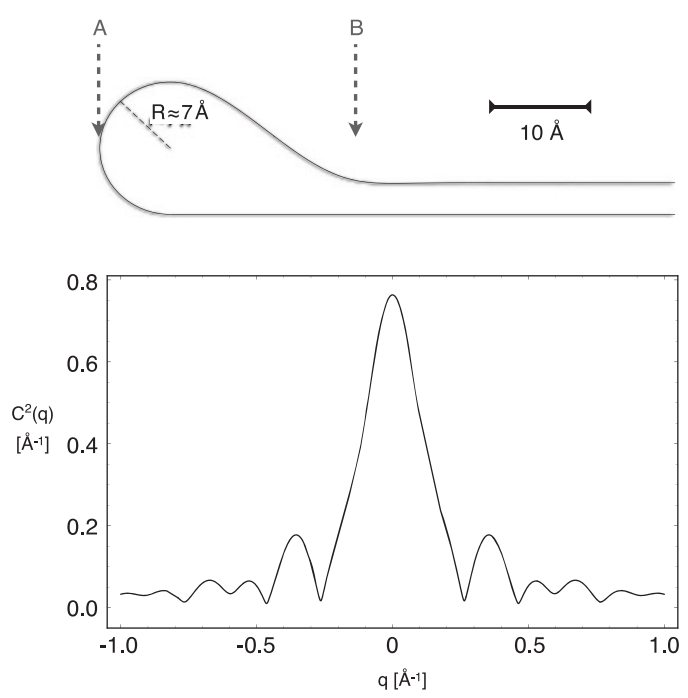

Figure 4. Numerical results for a phenomenological elastic model that includes van der Waals forces and the energy cost of curvature, as described in the main text. In the calculated profile of folded graphene (shown on top), the outer boundary of the bulb is assumed to be a half-circle while the shape of the inner boundary is optimized to minimize the total energy. Locations A and B refer to the marks on figure 1. The Fourier transform of the inverse squared radius of curvature $C^{2}=R^{-2}$ shows dominant contributions from wavelengths of 1 to $2 \mathrm{~nm}$.

that transitions from two effectively independent layers at the bulb to a true bilayer further away, since the G-band linewidth of few-layered graphene flakes increases with the number of layers. For skewed folding, the G-band linewidth decreases from 10 to $9 \mathrm{~cm}^{-1}$ across the fold. This opposite trend is also anticipated, since the G-band of a skewed bilayer is narrower even than that of a single layer, due to the very weak coupling between the two sheets of a skewed bilayer and the ability of each layer to half-shield the other from any extraneous adsorbates [9]. Thus the variations in the G-band linewidth across the fold provide independent evidence of bulbs at the folds, i.e. regions where the upper and lower portions of the sheet are lifted away from each other.

Whereas the G-band intensity increases linearly across the fold and then saturates in a manner consistent with a scattering channel that is dominated by interior-derived contributions, the D-band intensity for both commensurate and skewed systems reaches a maximum $a t$ the fold, with a width comparable to that of the laser spot: the bulb-induced D-band is apparently well confined to the immediate vicinity of the fold. For the skewed fold, the D-band does not go to zero in the sample interior. This remnant intensity comes from the so-called I-band, which is associated with interlayer coupling in bilayers [9] that have a skewed (possibly incommensurate) stacking between the upper and lower layers. The maximal D-band intensities for commensurate and skewed systems, normalized to their respective maximal G-band intensities, are 0.07 and 0.16 , respectively. Removing the contribution from the I-band in the flake interior, the effective intensity of the bulb-derived D-band in the skewed fold is about 0.12 , still significantly stronger than for the commensurate fold. This discrepancy can be ascribed to differing crystallographic orientations for the folds in commensurate and skewed bilayers, as explained below.

What is the source of the D-band at the folded bulb? The Raman D-band near $\sim 1350 \mathrm{~cm}^{-1}$ in $\mathrm{sp}^{2}$ carbons arises from a double-resonant (DR) Raman scattering process that is activated by the presence of a translation-symmetry-breaking inhomogeneity, typically structural disorder [14-16]. Double resonance has four steps: an incoming photon excites an electron-hole pair near the K point; the excited electron then scatters via an LO phonon to the vicinity of $\mathrm{K}^{\prime}$, followed by a disorder-induced elastic backscatter to $K$ [17] and a recombination of the electron and hole, with the emission of a photon. The order of the second and third steps may be reversed. We considered three possible sources for the observed D-band: discontinuity of a substrateinduced potential at a line of separation from the substrate, inhomogeneous curvature at the fold, or the loss of interlayer interaction as the bilayer separates into the bulb.

We exclude the first option by noting that Raman measurements performed on graphene suspended over a trench (not shown) do not see a D-band at the edge of the trench, where graphene abruptly separates from the substrate. To study the second possibility, inhomogeneous curvature (i.e. a local mean curvature whose magnitude changes as one traverses a bulb on a path perpendicular to the folded edge), we first find the bulb's shape using a phenomenological elastic model that takes into account van der Waals forces and the energy cost of curvature, similar to that already performed for collapsed carbon nanotubes [18, 19]. For simplicity, we assume that the lower part of the bulb is held flat to the substrate, terminating in a half-circle, as in figure 4(a). The remainder of the bulb's shape is optimized, as constrained by this boundary condition, to minimize the sum of the elastic and van der Waals energies. The resulting bulb cross-section has a radius of curvature of $7.0 \AA$ and a bulb width (measured along the upper layer) of about $3 \mathrm{~nm}$, which is consistent with the width observed by AFM. To estimate the strength of elastic scattering from the inhomogeneous curvature of the bulb, we employ a tight-binding formalism with wavefunctions $\psi_{k}(\vec{x})=\sum_{\sigma} \frac{C_{k}^{\sigma}}{\sqrt{N}} \sum_{R} \mathrm{e}^{\mathrm{i} k \cdot R} \varphi_{\sigma}(\vec{x}-\vec{R})$, where $\varphi_{\sigma}(\vec{x}-\vec{R})$ is an atomic orbital centered in the unit cell $\vec{R}$ on site $\sigma=$ $A, B$. The vector $C^{\sigma}$ and the energy bands are found [2] by diagonalizing the $2 \times 2$ matrix $\left(\begin{array}{cc}\varepsilon_{2 p} & f(k) \\ f^{*}(k) & \varepsilon_{2 p}\end{array}\right)$ with $f$ describing the intersite hopping. The matrix $\Delta H$ describing the scattering from curvature is

$$
\left\langle\psi_{k+q}|\Delta H| \psi_{k}\right\rangle=\vec{C}_{k+q}^{\perp}\left(\begin{array}{cc}
0 & \Delta f(q, k) \\
\Delta f^{*}(q, k) & 0
\end{array}\right) \vec{C}_{k},
$$

where

$$
\Delta f(q, k)=\frac{1}{N} \sum_{R, n} \mathrm{e}^{\mathrm{i} k \cdot e_{n}} \mathrm{e}^{\mathrm{i} q \cdot R} \Delta t_{n}(\vec{R})
$$

and $\Delta t_{i}(\vec{R})=\left\langle\varphi_{A}(\vec{x}-\vec{R})|\Delta H| \varphi_{B_{i}}(\vec{x}-\vec{R})\right\rangle$ represents the change in the hopping integral due to curvature for a bond directed along unit vector $e_{i}$. For uniform curvature, equation (2) returns $\Delta f(q, k) \propto \delta_{q, 0}$ and thus no scattering, but just an energy shift. A non-zero curvature gradient breaks translation invariance and thus can cause the elastic scattering 
needed for double resonance. Assuming no curvature gradient parallel to the folded edge, we obtain in the continuum limit:

$$
\Delta f(q, k)=\delta_{q_{y}, 0} \frac{1}{L} \sum_{n=1 \cdots 3} \mathrm{e}^{\mathrm{i} k \cdot e_{n}} \widetilde{\Delta t_{n}}\left(q_{x}\right),
$$

where $L$ is a normalization factor (i.e. the distance from the fold within which the electron is excited by the laser) and $\widetilde{\Delta t}_{n}\left(q_{x}\right)=\int \Delta t_{n}(x) \mathrm{e}^{\mathrm{i} q x} \mathrm{~d} x$ is the Fourier transform of the hopping integral for the $e_{n}$ bond, where $y$ is along the fold and $x$ perpendicular to it. Assuming that $\frac{\Delta t}{t} \approx-3 \frac{e^{2}}{R^{2}}$, where $e$ is the carbon-carbon bond length [20], we estimate the Fourier transform of the scattering Hamiltonian in the elastic model as $\Delta f / t \approx 10^{-1} e / L$. Unlike the physical edge of a graphene flake, which may be ragged due to irregularities in edge termination, the smooth and gentle elastic modulation of a fold should define a nearly ideal one-dimensional scattering potential. D-band scattering from physical sheet edges at the surface of bulk graphite shows clear evidence of edge roughness (i.e. wavevector non-conservation parallel to the edge) [21]. In contrast, elastic scattering from the folded bulb in the double-resonant process should occur along a welldefined direction in reciprocal space. The delta function $\delta_{q_{y}, 0}$ in equation (3) enforces this specular scattering. For example, it implies a reduced D-band intensity for folds along a zig-zag direction, which is an unfavorable orientation of the Brillouin zone for double-resonant scattering [21].

We now estimate $\Delta f / t$ for the remaining candidate mechanism, i.e. the loss of interlayer interaction where the two layers lift away from each other at the bulb. The experimental observation of comparable signals from commensurate and skewed folds argues strongly against such a lift-off mechanism, since the two layers of a skewed fold have a very weak interlayer electronic interaction [9, 22, 23]. Nevertheless, we estimate the relative strength of a lift-off mechanism for a commensurate fold, wherein the electronic states are coherent between layers so that the bulb is effectively an edge where the interlayer interaction terminates. Although $\mathrm{AB}$ stacking (i.e. zig-zag folds) gives a large interlayer hopping integral $(\sim 0.4 \mathrm{eV})$, double resonance is suppressed for zig-zag folds, as mentioned above, due to the unfavorable orientation of the Brillouin zone relative to the scattering potential from the folded edge. Armchair folding does not suffer from this orientation suppression, but its interlayer hopping integral is smaller $(\sim 70 \mathrm{meV})$. A tight-binding calculation then returns $\Delta f / t \approx 0.07 /(3 q L) \approx 0.01 e / L$, which is an order of magnitude smaller than the effect arising from inhomogeneous curvature. The large difference in the magnitudes of these two effects is likely to be robust against the variations in interlayer registry that could result from small shifts or shears of one layer relative to the other around the bulb. Having ruled out substrate lift-off and loss of interlayer interaction as possible mechanisms, we conclude that the fold-induced D-band arises from the spatial variation in the sheet curvature around the bulb.

Does the orientation of the fold relative to the underlying graphene lattice affect the double-resonant D-band response? Since the phase space available for double-resonant D-band scattering strongly peaks for phonon wavevectors along the direction that connects $\mathrm{K}$ and $\mathrm{K}^{\prime}$, armchair oriented folds (i.e. where a row of atoms moving along the fold assumes the armchair configuration) should support the strongest doubleresonant D-band. Folds along different crystallographic directions select different directions within the graphene Brillouin zone for specular scattering off the fold and hence support double resonance for phonon wavevectors of different magnitudes. Ideal, smooth folds close enough to the zigzag orientation should not support a double-resonant Dband at all for laser energies below a threshold. Therefore folds at different orientations should produce different Dband frequencies and intensities. The peak frequency for the commensurate fold in table $1,1344 \mathrm{~cm}^{-1}$, is similar to that from an edge of monolayer graphene [24] (but this does not necessarily imply that the edge and fold share a similar crystallographic orientation, since the edge in question might be ragged). As anticipated from the analysis above, the peak D-band frequency for the skewed fold is significantly different: $1351 \mathrm{~cm}^{-1}$. The narrowness of the fold-derived Dband provides additional support that scattering from the fold is specular, i.e. sharp in reciprocal space.

Variation of the incident polarization provides further evidence that the graphene fold is a nearly ideal onedimensional scatterer. Raman photon absorption and emission matrix elements are proportional to the cross product of the polarization vector and the electron wavevector measured relative to $\mathrm{K} / \mathrm{K}^{\prime}$ [25]. We control the incident polarization by rotating a half-wave plate with the laser spot remaining focused at the same point on the sample. Figures 3(c) and (d) plot the polarization dependence of the D-band from commensurate and skewed folds, with $\theta$ measuring the angle between the bulb edge and the incident (or scattered) electric field. We find nearly ideal $\cos ^{4} \theta$ behavior (factors of $\cos ^{2} \theta$ come from both photon absorption and emission [24]) with a very low residual signal at $\theta=90^{\circ}$. The low residual implies that scattering from the fold is nearly specular, i.e. the fold is straight and smooth in real space. The lack of a phase shift in $\theta$ suggests that the wavevectors of the intermediate electronic states (measured relative to $\mathrm{K} / \mathrm{K}^{\prime}$ ) are perpendicular to the fold direction, i.e. the fold is close to armchair orientation. In contrast, the D-band polarization response from armchair edges (measured at the surface of bulk graphite) shows a significant angle-independent background, which may be due to a ragged edge with non-specular reflection [21]. The polarization response for the D-band of a skewed fold is less definitive, since it has an additional polarizationindependent [9] contribution coming from I-band scattering in the bilayer interior. Hence we fit it to a constant plus $\cos ^{4} \theta$. Note that this analysis of the polarization in terms of the microscopic Raman matrix elements does not account for any modulation of the Raman response by larger-scale geometrical depolarization (i.e. antenna) effects due to the fold.

Does the experimental observation of commensurate versus skewed stacking in the bilayer interior constrain the possible fold orientations that produce these bilayers? Unfortunately, no. For graphene sheets with perfect shear rigidity, the zig-zag fold is the only orientation that supports the 
lowest-period commensurate stacking around a fold (i.e. AB stacking). However, this does not imply that commensurately folded bilayers always come from zig-zag folds (for which specular double-resonant scattering would be suppressed, in contradiction to our observations), since a very gentle elastic shear deformation over a distance comparable to the laser spot size could relax, for example, a D-band-generating armchair fold into $\mathrm{AB}$ registry. (A perfectly shear-rigid armchair fold would also produce a commensurate bilayer, albeit not of the lowest-energy $\mathrm{AB}$ variety.) Unfortunately, the required elastic deformation is very gentle-a fraction of a bond length over hundreds of angstroms - and does not necessarily involve a commensurate-incommensurate transition. Hence it is not expected to generate an easily perceptible Raman signature. In a similar vein, skewed folds could have almost any orientation, even those very close to armchair or zig-zag.

In summary, we demonstrate that spatially varying curvature in the bulbs of a folded graphene sheet provides a new form of inhomogeneous scattering potential that can generate double-resonant D-band scattering in both commensurate and skewed (or incommensurate) folds. The gentle, long-wavelength nature of the elastic interactions that govern the geometry of the fold should generate ideal specular scattering at the interface unclouded by issues of ragged edge disorder that complicate the interpretation of D-band scattering from flake edges. The frequency and intensity of this fold-induced D-band depend on the orientation of the fold relative to the graphene lattice, whereas the strong polarization dependence of the Raman scattering from this one-dimensional defect apparently does not.

\section{Acknowledgments}

This work was supported by the NSF NIRT ECS-0609243. We acknowledge contribution of Ke Zou in acquiring AFM images.

\section{References}

[1] Dresselhaus M S, Dresselhaus G and Eklund P C 1996 Science of Fullerenes and Carbon Nanotubes (San Diego, CA: Academic)

[2] Saito R 1998 Physical Properties of Carbon Nanotubes (Singapore: World Scientific)
[3] Dresselhaus M S and Eklund P C 2000 Adv. Phys. 49 705-814

[4] Li X, Wang X, Zhang L, Lee S and Dai H 2008 Science 319 1229-32

[5] Cancado L G, Pimenta M A, Neves B R A, Medeiros-Ribeiro G, Enoki T, Kobayashi Y, Takai K, Fukui K, Dresselhaus M S, Saito R and Jorio A 2004 Phys. Rev. Lett. 93047403

[6] Cancado L G, Takai K, Enoki T, Endo M, Kim Y A, Mizusaki H, Jorio A, Coelho L N, Magalhaes-Paniago R and Pimenta M A 2006 Appl. Phys. Lett. 88163106

[7] Novoselov K S, Jiang D, Schedin F, Booth T J, Khotkevich V V, Morozov S V and Geim A K 2005 Proc. Natl Acad. Sci. 102 10451-3

[8] Poncharal P, Ayari A, Michel T and Sauvajol J L 2008 Phys. Rev. B 78113407

[9] Gupta A K, Tang Y, Crespi V H and Eklund P C 2010 arXiv:1005.3857

[10] Ni Z, Wang Y, Yu T, You Y and Shen Z 2008 Phys. Rev. B 77235403

[11] Ferrari A C, Meyer J C, Scardaci V, Casiraghi C, Lazzeri M, Mauri F, Piscanec S, Jiang D, Novoselov K S, Roth S and Geim A K 2006 Phys. Rev. Lett. 97187401

[12] Graf D, Molitor F, Ensslin K, Stampfer C, Jungen A, Hierold C and Wirtz L 2007 Nano Lett. 7 38-42

[13] Gupta A, Chen G, Joshi P, Tadigadapa S and Eklund P C 2006 Nano Lett. 6 2667-73

[14] Ferrari A C and Robertson J 2000 Phys. Rev. B 61 14095-107

[15] Thomsen C and Reich S 2000 Phys. Rev. Lett. 85 5214-7

[16] Saito R, Jorio A, Souza Filho A G, Dresselhaus G, Dresselhaus M S and Pimenta M A 2002 Phys. Rev. Lett. 88 7401-4

[17] Pimenta M A, Dresselhaus G, Dresselhaus M S, Cançado L G, Jorio A and Saito R 2007 Phys. Chem. Chem. Phys. 9 1276-91

[18] Qian D, Wagner G J, Liu W K, Yu M-F and Ruoff R S 2002 Appl. Mech. Rev. 55 495-533

[19] Chopra N G, Benedict L X, Crespi V H, Cohen M L, Louie S G and Zettl A 1995 Nature 377 135-8

[20] Guinea F, Katsnelson M I and Vozmediano M A H 2008 Phys. Rev. B 77075422

[21] Cançado L G, Pimenta M A, Neves B R A, Dantas M S S and Jorio A 2004 Phys. Rev. Lett. 93247401

[22] Latil S, Meunier V and Henrard L 2007 Phys. Rev. B 76201402

[23] Lopes dos Santos J M B, Peres N M R and Castro Neto A H 2007 Phys. Rev. Lett. 99256802

[24] Gupta A K, Russin T J, Gutierrez H R and Eklund P C 2009 ACS Nano 3 45-52

[25] Gruneis A, Saito R, Samsonidze G G, Kimura T, Pimenta M A, Jorio A, Filho A G S, Dresselhaus G and Dresselhaus M S 2003 Phys. Rev. B 67165402 